Neurosurg Focus 5(6):Article 1, 1998

\title{
Carotid endarterectomy: current indications for surgery
}

James J. Brennan, M.D., and Christopher M. Loftus, M.D.

Department of Neurosurgery, University of Oklahoma Health Sciences Center, Oklahoma City, Oklahoma

The study of carotid artery occlusive disease interventions can be divided clinically into the treatment of asymptomatic and symptomatic diseases. Clinical trials that have studied or are currently studying asymptomatic disease include: the Carotid Artery Stenosis with Asymptomatic Narrowing Operation Versus Aspirin study; the Mayo Asymptomatic Carotid Endarterectomy trial; Veterans Administration Cooperative Trial on Asymptomatic Carotid Stenosis; and the Asymptomatic Carotid Atherosclerosis Study. Trials for the treatment of symptomatic disease include: the North American Symptomatic Carotid Endarterectomy Trial; the European Carotid Surgery Trial; and the Veterans Administration Cooperative Study.

In the earliest trials conducted to study asymptomatic disease medical therapy was slightly favored; on close scrutiny these studies were flawed and the findings appeared to be equivocal. The more scientific and appropriate trial, which was ended due to ethical concerns, revealed a clear advantage in patients who underwent surgery for greater than $60 \%$ stenosis and when the surgical center demonstrated less than $3 \%$ surgical risks.

All trials studying symptomatic disease found a significant decrease in subsequent stroke when surgical intervention was performed. It is now judged that patients with greater than $50 \%$ stenosis receive significant benefit.

In this paper the authors review the data from all of these studies. They also review data for special circumstances, such as critical stenosis and patients with symptomatic and asymptomatic Hollenhorst plaques. It is their opinion that these data have allowed surgeons to make much more educated decisions when considering the treatment of patients with carotid artery occlusive disease.

Key Words * Asymptomatic Carotid Atherosclerosis Study * carotid artery surgery * carotid endarterectomy * European Carotid Surgery Trial * North American Symptomatic Carotid Endarterectomy Trial * stroke

Although the use of carotid endarterectomy (CEA) in the prevention of ischemic stroke was first described in 1954,[5] it was never well studied in randomized carotid artery studies until the past decade. 
Recent cooperative trials have been undertaken to determine the best indications for this procedure. In this paper we describe the results of these trials and present the current algorithm we use for treating patients with carotid artery occlusive disease.

Diseases of the carotid artery circulation can be clinically divided into two types: asymptomatic and symptomatic. A diagnosis of asymptomatic disease is indicated in patients with incidentally found carotid bruits, cerebrovascular symptoms referable to one carotid artery territory, and demonstration of an asymptomatic contralateral carotid artery stenosis or ulceration, and patients with auscultatory or radiographic evidence of carotid artery disease should be scheduled to undergo general or cardiac surgery. Two subgroups also included in this category are patients with asymptomatic Hollenhorst plaques and patients in whom serial carotid artery studies demonstrate increasing primary or recurrent stenosis.

A diagnosis of symptomatic disease is indicated in patients with classic transient ischemic attacks (TIAs), crescendo TIAs, and reversible ischemic neurological deficits. All of these symptoms may coexist with amaurosis fugax, hemimotor and/or hemisensory deficits, and/or disturbances in speech, and all vary in severity, frequency, and duration. Other symptoms included in this category are completed stroke, acute or subacute carotid artery occlusion (if neurological changes are noted), or stroke in evolution. Patients whose symptoms occur on the side of an occluded internal carotid artery, known as "stump syndrome," are also included in this category.

Since surgical intervention for carotid artery occlusive disease was first described many confusing and contradictory studies that described both asymptomatic and symptomatic treatments have been published. More recently, prospective cooperative studies have been completed that confirm that surgery has a role in the treatment of this disease. A review of these trials, however, including an understanding of which populations were studied and what conclusions can be reached, is imperative if these data are to be applied in an individual patient. It is also important to recognize that the acceptable surgical risk for elective CEAs should be less than 3\%. The American College of Physicians and the American Heart Association have developed guidelines that specify the acceptable surgical morbidity and mortality rates. If these guidelines are not met, the surgical benefit of performing CEA disappears, especially for patients with asymptomatic disease.

\section{COOPERATIVE TRIALS FOR ASYMPTOMATIC DISEASE}

Four major prospective cooperative trials evaluating surgical compared with nonsurgical management of carotid artery occlusive disease have been completed; the Carotid Artery Stenosis with Asymptomatic Narrowing Operation Versus Aspirin Study; the Mayo Asymptomatic Carotid Endarterectomy trial; the Veterans Administration Cooperative Trial on Asymptomatic Carotid Stenosis (VA CSP 167); and the Asymptomatic Carotid Atherosclerosis Study (ACAS). Another trial (the Asymptomatic Carotid Stenosis Trial) is still being conducted in Europe. Unlike the trials for symptomatic disease, these four trials, have yielded conflicting results.

The criterion for surgery in all studies has been the percentage of linear stenosis angiographically demonstrated in the cervical carotid artery. (Although patients in the medical arm of the ACAS could enter the trial without having undergone arteriography, it was required for all surgical patients.) Stenosis morphology and the presence of ulcerations were noted but they were not considered when the patients were randomized. 
The Carotid Artery Stenosis with Asymptomatic Narrowing Operation Versus Aspirin Trial was the first of these trials. The study was published in 1991[3] and the patients received medical therapy that included aspirin and dipyridamole; patients with a greater than $90 \%$ stenosis were excluded. This study reported no reduction in morbidity and mortality rates; however, it was flawed because crossover from the medical group to surgical group for progressive stenosis was allowed, and empirical surgery was performed in patients with more than $90 \%$ stenosis or with known contralateral occlusion. As later studies were published, this study has become somewhat discredited. A total of 410 patients with a linear stenosis of 50 to $90 \%$ were studied in this trial.

The Mayo Asymptomatic Carotid Endarterectomy Study was terminated early because patients in the surgical group, whose antiplatelet therapy was discontinued in the postoperative period, were placed at increased risk for a cardiac event.[11] No significant conclusions could be drawn from this study except for the importance of antiplatelet therapy in the treatment of patients with coronary artery disease. A total of 71 patients with a linear stenosis of 50 to $99 \%$ were studied in this group.

The results of the VA CSP 167 trial, published in 1992, demonstrated that surgical intervention reduced subsequent ipsilateral transient neurological events but not mortality or stroke rates.[9] This finding caused further debate regarding the benefit of surgery for asymptomatic lesions. A total of 444 men with greater than $50 \%$ stenosis were studied in this trial.

The largest, most recent, and most informative of all these studies is the ACAS, which was published in 1995.[7] Stenosis was graded by carotid artery duplex in all patients, but angiographic confirmation of disease was required only in those patients undergoing surgery. The primary outcome measure in this trial was the existence any stroke or death in the perioperative period and any ipsilateral stroke thereafter. Patients who received medical treatment had risk reduction measures with $325 \mathrm{mg} /$ day of aspirin. Patients assigned to the surgical group underwent the same medical protocol after surgery was performed.

After a median follow-up period of 2.7 years, the aggregate 5-year estimated risk for ipsilateral stroke or perioperative stroke or death was $5.1 \%$ in patients who underwent surgery and $11.0 \%$ in patients treated medically. Surgical intervention was shown to reduce the estimated 5-year risk of stroke by $1 \%$ per year, with a combined perioperative morbidity and mortality rate of less than $3 \%$ required for the surgical center. This benefit was significant for the overall group and for the male study population subgroup, but no significance was found when women were analyzed as a discrete population subgroup. This outcome may be due to the early termination of the study (average follow-up period of 2.7 years) when ethical concerns arose given the obvious outcome improvement in patients who underwent surgery. A total of 1659 patients at 39 centers with 60 to $99 \%$ stenosis participated in this trial.

Unfortunately and somewhat counterintuitively, the asymptomatic randomized studies, notably ACAS, could not stratify stroke risk from carotid artery occlusive disease by the percentage of stenosis. This increased risk (higher risk with higher degree of stenosis) was only proven in the symptomatic cooperative trials. Some evidence for increased risk with high-grade asymptomatic stenosis was shown when the European Carotid Surgery Trial (ECST), a trial that studied symptomatic disease, attempted to stratify by the percentage of stenosis the contralateral asymptomatic arteries.[6] The ECST is not directly comparable to the ACAS because it was retrospective, different measurement techniques were used to determine the percentage of stenosis, and the studies were of different lengths. The ECST showed a definite, although not definitive risk for patients with greater than $70 \%$ stenosis and significant surgical 
benefits in patients with 80 to $90 \%$ and 90 to $99 \%$ stenosis.

Overall, a review of the clinical trials that were conducted to determine the effectiveness of surgery for asymptomatic carotid occlusive disease reveals that early studies, with some flaws, did not advocate surgical intervention. However, the more directed later study, ACAS, revealed that a significant benefit was derived from surgical intervention for asymptomatic patients with greater than $60 \%$ stenosis (when determined angiographically by the ACAS measurement protocol). Although some subgroups of patients have not been well stratified, other trials, including a randomized European asymptomatic trial, will hopefully resolve these issues.[8]

\section{COOPERATIVE TRIALS FOR SYMPTOMATIC DISEASE}

Three major clinical trials have been completed that sought to determine the benefit of surgery compared with medical therapy in the treatment of patients with carotid artery occlusive disease. These include the North American Symptomatic Carotid Endarterectomy Trial (NASCET),[13] ECST, and VA CSP 309.[10] The results of these studies uniformly indicated that surgery for symptomatic patients with greater than $70 \%$ stenosis attained a significant benefit compared with medical therapy. More recently the NASCET has proven that symptomatic patients with $50 \%$ or greater stenosis benefit from surgical intervention.

First published in 1991, the NASCET data revealed a significant reduction in the overall risk of stroke within 2 years postsurgery. Although there was a slight increase in the risk of stroke within 30 days postsurgery, this risk was far outweighed by the overall benefit. These results, obtained in patients with 70 to $99 \%$ stenosis, were based on an assumed perioperative stroke rate of less than $6 \%$. The benefits in patients were as follows: $17 \%$ for ipsilateral stroke; $15 \%$ for all strokes; $16.5 \%$ for the combined outcome of all strokes and death; $10.6 \%$ for major ipsilateral stroke; $9.4 \%$ for all major strokes; and $10.1 \%$ for major stroke and death. The study included 659 patients who presented with TIAs or a nondisabling stroke that had been sustained within the prior 120 days and in whom angiographic evidence of 70 to $99 \%$ stenosis was obtained.

More recently, patients in a NASCET "moderate" stenosis group (30-59\% stenosis) underwent further stratification, and benefit from surgery in patients with 50 to $69 \%$ stenosis was reported.[1] This benefit was most apparent for men, nondiabetic patients, and patients with hemispheric (not retinal) TIAs. An overall benefit of a $29 \%$ risk reduction was established, with the estimation that 15 CEA procedures would be needed to prevent one stroke.

Also released in 1991, the ECST symptomatic carotid artery data were based on 1152 patients enrolled in the trial. This study stratified patients into three groups: 0 to $29 \%, 30$ to $69 \%$, and 70 to $99 \%$ carotid artery stenosis. Results from ECST did not demonstrate a surgical benefit for patients in the moderate (30-69\%) stenosis category. Unfortunately, the measuring system was not as stringent as that used for the NASCET, and it is believed that this stratification was not qualitatively equal to that of the NASCET. This means that a patient who was considered to have moderate stenosis in ECST may not even have qualified for inclusion in the trial. Therefore, the negative for moderate stenosis is controversial, and it is probable that the NASCET data, having been subjected to a more rigorous method of measurement, will be definitive in guiding the management of patients with moderate stenosis.

The VA CSP 309 symptomatic carotid artery trial was a smaller trial and had a shorter follow-up period because it was prematurely discontinued when the NASCET and ECST data were released (these trials 
answered the main hypothesis of VA CSP 309). Despite the small number of patients (189), the data from this study confirmed a surgical benefit for patients with 70 to $99 \%$ stenosis, and there was a demonstrable benefit for patients with moderate stenosis of $50 \%$ or greater as well.

These data overwhelmingly support the use of surgery for the treatment of patients with 70 to $99 \%$ carotid artery stenosis. The more recent NASCET data, as well as these obtained in the earlier VA SCP 309 trial, also confirm that selected patients with 50 to $69 \%$ stenosis would benefit from surgical treatment.

\section{ISSUES NOT ADDRESSED BY THE CLINICAL TRIALS}

Certain patients with carotid artery occlusive disease cannot be generalized to an asymptomatic or a symptomatic trial and, as such, were not studied in the aforementioned trials. These include patients with hemodynamically critical stenosis (especially recurrent disease) and Hollenhorst plaques.

\section{Critical Stenosis and Recurrent Disease}

Critical stenosis may be documented in the asymptomatic patient by noninvasive studies, magnetic resonance angiography, or angiography. Studies reveal that reductions in cerebral blood flow begin to occur when stenosis is at 75 to $84 \%$, and it is believed that a residual lumen diameter of 2-mm represents at least a $70 \%$ stenosis.[14] Therefore a stenosis of less than $2 \mathrm{~mm}$ residual lumen or greater than $80 \%$ stenosis is required for the "critical stenosis" to be hemodynamically significant. When and if these asymptomatic patients required primary surgery was widely debated prior to the ACAS study. It is now our opinion that all of these patients should be categorized as asymptomatic patients with greater than $60 \%$ stenosis; therefore, patients with critical stenosis are best treated by undergoing surgical reconstruction.

The issue is less clear for patients in whom asymptomatic carotid artery disease recurs. There is a small but finite incidence of recurrent carotid artery stenosis that occurs after primary CEA. Most authors cite a symptomatic recurrence rate of approximately 4 to $5 \%$, and in one study in which noninvasive follow-up evaluation was performed after carotid artery surgery, a $4.8 \%$ recurrence rate of symptomatic carotid artery restenosis was documented, with an additional 6.6\% silent restenosis rate.[16] The Mayo series has cited a lower figure after performing patchgraft repair (0.05 to $1.0 \%$ per year).[17]

Aside from technical inadequacies, it has been difficult to identify the risk factors associated with recurrent carotid stenosis, although the continuation of cigarette smoking post-CEA proved to be a significant risk factor in one study; however, hypertension, diabetes mellitus, family history, lipid studies, aspirin use, and coronary disease were not found to be significant risk factors.[4]

Reoperation for carotid stenosis is a technically difficult procedure and is associated with significantly higher risks than primary CEA. Piepgras and Sundt[17] have documented a risk of complications rate of $10.5 \%$, which is four times higher than their customary rate.[16] In our institution, reoperation for carotid stenosis is considered in patients who present with angiographically proven disease and classical neurological symptoms referable to the appropriate artery. Until the ACAS data were released, we had been unwilling to assume this surgical risk in asymptomatic patients and, because of this, have not routinely performed noninvasive studies in our patients beyond the 1st year if they remain clinically stable. Other practioners with great experience in this field, however, do believe that the presence of changing bruits or rapidly progressive stenosis justifies surgical intervention.[17] It remains unclear whether the surgical benefits shown by ACAS for primary cases can be extrapolated to recommend 
reconstruction for patients with asymptomatic recurrent disease. It is our policy to offer surgery to patients with recurrent neurological symptoms that are associated with significant recurrent stenosis, and we have begun to perform surgery in patients with critical asymptomatic recurrence as well. It is unlikely that any cooperative trial will ever be able to resolve these questions in a randomized fashion.

\section{Hollenhorst Plaques}

Retinal cholesterol emboli, or Hollenhorst plaques may be discovered as symptomatic or asymptomatic lesions. It has been demonstrated in symptomatic patients that their survival is reduced by $13 \%$ over a prolonged period; the greatest risk factor is peripheral and cardiovascular disease. Therefore it is at least warranted to evaluate these patients for carotid artery occlusive disease.[15]

Patients with asymptomatic Hollenhorst plaques form a much smaller group and yet pose a greater diagnostic dilemma. In a study of 70 men with asymptomatic Hollenhorst plaques, although there was no significant increase in the number of patients with ipsilateral artery carotid occlusive disease, there was a significant increase in carotid artery occlusive diseases when both the ipsilateral and contralateral carotid arteries were studied.[2]

The studies of patients with Hollenhorst plaques have indicated that in these patients noninvasive evaluation for carotid artery occlusive disease is definitely warranted; however, whether or not surgical intervention should be performed must be based on the criteria outlined in these studies on symptomatic and asymptomatic patients.

\section{Overview Treatment of the Patient With Carotid Artery Occlusive Disease}

Whereas asymptomatic patients will usually present as outpatients in whom noninvasive studies have or have not been obtained, symptomatic patients more often present as inpatients and may have undergone more definitive testing.

When no studies are available, we recommend that an initial carotid artery duplex scan be obtained to establish diagnosis. If the imaging study is suggestive of carotid artery occlusive disease, further medical evaluation is undertaken to treat any conditions (such as cardiac disease) that could preclude the patient from surgery. If the study is suggestive of critical stenosis, the evaluation is expedited. The patient undergoes antiplatelet therapy (preferably enteric, coated aspirin) if asymptomatic or mildly symptomatic (patients with significant symptoms that warrant admission for urgent evaluation and therapy). The antiplatelet therapy is continued up until surgery.

When the patient is deemed an acceptable surgical candidate a complete angiographic study is performed. It does not seem likely that carotid artery duplex scan alone will be accepted as the diagnostic measure in the surgical treatment of carotid artery occlusive disease; however, with the improvement in magnetic resonance angiography, this may soon be accepted for a preoperative evaluation. It remains our preference to perform formal and complete angiography, including selective injection of both carotid arteries and an arch arteriogram. Attention to intracranial cross filling is also important, and we insist on biplane cervical angiography in every case to determine the relationship of the external and internal carotid arteries. When digital angiography is performed, as is currently common in our center, we require that the arterial image be superimposed on a bone image so that we can ascertain the height of the carotid artery bulb in relation to the cervical spine, the angle of the mandible, and the hyoid bone.

Once the angiogram is obtained and if significant carotid artery disease is demonstrated, we discuss the 
indications (including cooperative trial data) and the risk/benefit ratio with the patient. A complete history is obtained, a physical examination is performed (with detailed neurological examination), and surgery is scheduled. Patients with a significant smoking history are urged to quit, and an appointment to a smoking cessation clinic is offered. Surgery is usually scheduled within 1 or 2 weeks.

Patients who present with crescendo TIAs undergo intravenous heparin therapy, and an arteriogram is obtained immediately. If a surgical lesion is identified (as is nearly always the case), surgery is performed within 24 hours while the patient recieves anticoagulation medication.

\section{References}

1. Barnett H, Taylor D, Eliasziw M, et al: Benefit of carotid endarterectomy in patients with symptomatic moderate or severe stenosis. N Engl J Med 339:1415-1440, 1998

2. Bruno A, Russell PW, Jones WL, et al: Concomitants of asymptomatic retinal cholesterol emboli. Stroke 23:900-902, 1992

3. CASANOVA Study Group: Carotid surgery versus medical therapy in asymptomatic carotid stenosis. Stroke 22:1229-1235, 1991

4. Clagett GP, Rich NM, McDonald PT, et al: Etiologic factors for recurrent carotid artery stenosis. Surgery 2:313-318, 1983

5. Eastcott H, Pickering G, Rob CG: Reconstruction of internal carotid artery in a patient with intermittent attacks of hemiplegia. Lancet 2:944-946, 1954

6. European Carotid Trialists Collaborative Group: risk of stroke in the distribution of an asymptomatic carotid artery. Lancet 345:209-212, 1995

7. Executive Committee for the Asymptomatic Carotid Atherosclerosis Study: endarterectomy for asymptomatic carotid artery stenosis. JAMA 273:1421-1428, 1995

8. Halliday AW, Thomas D, Mansfield A: The asymptomatic carotid surgery trial (ACST). Rationale and design. Eur J Vasc Surg 8:703-710, 1994

9. Hobson RW II, Weiss DG, Fields WS, et al: Efficacy of carotid endarterectomy for asymptomatic carotid stenosis. New Engl J Med 328:221-227, 1993

10. Mayberg MR, Wilson SE, Yatsu F, et al: Carotid endarterectomy and prevention of cerebral ischemia in symptomatic carotid stenosis. JAMA 266:3289-3294, 1991

11. Mayo Asymptomatic Carotid Endarterectomy Study Group: results of a randomized controlled trial of carotid endarterectomy for asymptomatic carotid stenosis. Mayo Clin Proc 67:513-518, 1992

12. MRC European Carotid Surgery Trial: interim results for symptomatic patients with severe (70-99\%) or with mild (0-29\%) carotid stenosis. Lancet 337:1235-1243, 1991

13. North American Symptomatic Carotid Endarterectomy Trial Collaborators: beneficial effect of carotid endarterectomy in symptomatic patients with high grade stenosis. N Engl J Med 325:445-453, 1991 
14. Ojemann RG, Crowell RM, Robertson G, et al: Surgical treatment of extracranial carotid occlusive disease. Clin Neurosurg 22:214-263, 1975

15. Pfaffenbach DD, Hollenhorst RW. Morbidity and survivorship of patients with embolic cholesterol crystals in the ocular fundus. Am J Ophthalmol 75:66-72, 1973

16. Salvian A, Baker JD, Machleder HI: Cause and noninvasive detection of restenosis after endarterectomy. Am J Surg 146:29-34, 1983

17. Sundt TM Jr, Whisnomt JF, Houser OW, et al: Prospective study of the effectiveness and durability of carotid endarterectomy. Mayo Clin Proc 65:625-635, 1990

Manuscript received November 30, 1998.

Accepted in final form December 10, 1998.

Address reprint requests to: Christopher M. Loftus, M.D., Department of Neurosurgery, University of Oklahoma Health Sciences Center, 711 Stanton L. Young Boulevard, Suite 206, Oklahoma City, Oklahoma 73104. email: chris-loftus@ouhsc.edu. 